\title{
Quality Assurance and Quality Control of an Intensive Care Unit Picture Archiving and Communication System
}

\author{
Douglas M. Tucker and Michael McEachern
}

\begin{abstract}
Most radiology departments have established quality assurance $(\mathrm{QA})$ and quality control $(\mathrm{QC})$ programs for conventional film-based image management systems. At many institutions, digital image management systems, or picture archiving and communication systems (PACS), are replacing part or all of the film management system. In these situations, it is important to control the quality of the digital images that are produced. The observed frequency of eight types of image-related errors occurring on an image viewing station located in a medical intensive care unit is reported. Images on the viewing station were checked for 12 consecutive weeks. Film images available in the radiology reading room and digital images on the viewing station were compared with a list of completed examinations produced by the radiological information system. Overall, 1,082 patient examinations were encountered. Seventy-six images $17.02 \%$ of all images) were observed with errors. In addition, four previously unencountered types of errors were observed in 11 images ( $1.01 \%$ of all images). The majority of the errors are attributed to interfaces either between information systems or between the PACS and the user. It is concluded that QA-QC procedures are necessary for PACS, and that good interfaces, both between information systems and between humans and computer systems, are essential for successful PACS implementations.
\end{abstract}

Copyright $\odot 1995$ by W.B. Saunders Company

KEY WORDS: picture archiving and communication systems (PACS), intensive care unit (ICU), quality control, quality assurance

$\mathbf{T}$ HE GOAL of a quality assurance (QA) program in diagnostic radiology is to guarantee the delivery of timely and accurate information. Secondary goals include reducing patient radiation exposure risk, discomfort, and cost to the patient and community. ${ }^{1}$ According to the National Council on Radiation Protection (NCRP), ${ }^{2}$ quality control (QC) is

a series of distinct technical procedures which ensure the production of a satisfactory product. Four major steps are involved.

From the Department of Radiology, University of Alabama Hospital and Clinics, Birmingham, $A L$.

Address reprint requests to Douglas M. Tucker, PhD, Department of Radiology, 619 S 19th St, Birmingham, AL 35233.

Copyright $\odot 1995$ by W.B. Saunders Company

0897-1889/95/0804-0003\$3.00/0
- Acceptance testing detection of defects in equipment that is newly installed or has undergone major repair.

- Establishment of baseline performance of equipment.

- Diagnosis of changes in equipment performance before they become radiologically apparent.

- Verification of correction of causes of deterioration in equipment performance.

In this paper, results of a QA program established to monitor the performance of an intensive care unit (ICU) picture archiving and communication system (PACS) are reported.

At many institutions, PACS, or limitedfunction PACS (mini-PACS), ${ }^{3}$ are being implemented. Examples of mini-PACS include centralized laser film printers, ultrasound and nuclear medicine imaging networks, and critical care PACS ${ }^{4-8}$ Because of the immature state of the technology, information concerning quality assurance or quality control of PACS is scarce. ${ }^{9 \cdot 12}$ There are few reports that outline acceptance testing procedures for PACS components ${ }^{13}$ (workstation monitors, for example). These reports do not address issues related to the quality of information managed by the PACS. Guidelines concerning the implementation of a QA-QC program for information (images, reports, etc) managed by a PACS are also scarce. ${ }^{14}$ To our knowledge, there is no literature reporting the results of comprehensive PACS QA-QC programs.

At our institution, an ICU PACS ${ }^{15}$ has been developed. Storage phosphor computed radiography (CR) is used for direct digital image capture of bedside radiographs. ${ }^{16} \mathrm{~A}$ technologist substitutes a CR storage phosphor plate for a conventional screen-film receptor during routine bedside radiographic examinations. CR plates are processed using one of two Fuji $\mathrm{ACl}+\mathrm{s}$ (Fuji Medical Systems, Stamford, CT) located in the radiology department. Each image is identified by a flashcard and by the manual entry of an order-specific requisition number. Hard-copy film is produced for radiologic interpretation, and the corresponding digital data is captured by the ICU PACS system. 
Image processing, storage, distribution and viewing are performed by a network of UNIX (C) workstations running software developed inhouse. Images are transferred to viewing stations located in the patient-care areas. Radiologic interpretations, when completed, are retrieved from the radiological information system (RIS) and associated with each image. Two backup copies of each image and interpretations are stored in separate image storage systems. Images and interpretations can be viewed by personnel in the care-delivery area on image viewing stations. Images are kept in the viewing station and backup storage for the duration of the patient stay; image and report data are removed within 24 hours of patient discharge. Every portable chest and abdomen examination performed at our institution is processed by the ICU-PACS. Currently, five viewing stations are installed in patient care areas (Table 1).

Baseline performance levels for the quality of radiologic information delivered to remote viewing stations need to be established. Goals of this study were to identify and establish the frequency of observation for parameters indicative of quality of information available on a viewing station. Ideally, every image should be properly identified, oriented correctly, transmitted to the proper viewing station and have the radiologic interpretation available. Parameters identified in this study relate to image availability, timeliness of image and report delivery, and accuracy. Issues related to PACS hardware (monitors, digitizers, etc) quality control are not addressed.

\section{MATERIALS AND METHODS}

In this study, eight types of errors related to the quality of information delivered to an image viewing station in a medical ICU (MICU) are identified. These parameters are listed in Table 2. During a 12-week period beginning in September 1994 and ending in December 1994, data were collected to evaluate the magnitude of these errors. Data were collected Monday through Friday, excluding holidays.

Table 1. Locations of Image Viewing Station in ICU PACS

\begin{tabular}{lccc}
\hline \multicolumn{1}{c}{ Location } & $\begin{array}{c}\text { No. Beds } \\
\text { Served }\end{array}$ & $\begin{array}{c}\text { Avg No. } \\
\text { Patients }\end{array}$ & $\begin{array}{c}\text { Avg No. } \\
\text { Exams }\end{array}$ \\
\hline Medical ICU & 12 & 11.14 & 12.88 \\
Cardiac ICU & 20 & 14.10 & 25.79 \\
Surgical ICU & 18 & 15.10 & 23.14 \\
Neuroscience ICU & 21 & 18.50 & 20.75 \\
Coronary CU & 20 & 13.57 & 8.09 \\
\hline
\end{tabular}

Images associated with patient examinations performed on weekends and holidays were included in this study; these images were examined on the next work day.

A standard procedure for data collection was established. To acquire data, a list of completed bedside examinations performed on patients in the MICU over the previous 2.5 days was generated. This information was retrieved from reports produced by the RIS each day between 6:30 AM and 7:00 AM. Similarly, a list of images available on the viewing station in the MICU was generated at 7:00 AM.

The list of completed examinations was verified by reviewing the film images of MICU patients available in the radiology department's reading room. Differences between the RIS-generated list of completed orders and films available in the reading room were recorded. Discrepancies between the patient identification on the film image and order request were also recorded. The RIS-generated list of completed examinations and the list of images available on the MICU viewing station were similarly compared. Missing images and images inadvertently sent to the viewing station were recorded. All new images (those not reviewed as part of the QC activity since the previous check of the viewing station) were displayed and checked for correct identification and proper orientation. Images acquired on the previous day were checked for the presence or absence of the radiologic interpretation. The area surrounding the viewing station was searched for film images. Films that were found were checked against the list of films missing from the radiology reading room. All discrepancies were recorded.

\section{RESULTS}

During the 12-week period of the survey, 1,082 bedside radiologic examinations were conducted on MICU patients. The MICU has 12 beds. The average occupancy during the study was 11.14 patients per day with 12.88 radiographic examinations performed per day. During the study, 76 errors $(7.02 \%$ of all examinations) were observed. The errors and frequency of occurrence are listed in Table 2.

During the study period, four previously undetected types of errors were discovered. Table 3 lists these additional errors and their frequency of occurrence. These errors were (1) improper image processing (a chest examination was processed as an abdomen); (2) failure of the CR system to properly process a patient identifying flashcard; (3) incorrect patient census information leading to premature removal of patient images from the viewing station, and (4) repeated examinations (positioning errors) transmitted to viewing station. In total, 11 additional occurrences of such errors (1.01\% of all examinations) were observed during the course of the study.

Each type of error has been classified accord- 
Table 2. Image Quality Parameters

\begin{tabular}{|c|c|c|c|c|}
\hline Parameter & Frequency & Impact on Information & Uniqueness to PACS & Cause \\
\hline Incorrect order number & 7 & $S$ & Yes & Miskeyed order number \\
\hline Wrong patient & 2 & S & No & $\begin{array}{l}\text { (1) Miskeyed order number } \\
\text { (2) RIS-HIS failure }\end{array}$ \\
\hline Wrong flash card & 2 & S & No & Technologist error \\
\hline Lost image & 14 & L & No & $\begin{array}{l}\text { (1) Miskeyed order number } \\
\text { (2) RIS-HIS failure } \\
\text { (3) Hardware failure }\end{array}$ \\
\hline No order number & 10 & $L$ & No & RIS-HIS failure \\
\hline No diagnosis & 18 & L & No & $\begin{array}{l}\text { (1) No image interpretation } \\
\text { (2) RIS-HIS failure }\end{array}$ \\
\hline Orientation & 14 & 1 & No & Technologist $\mathrm{OC}$ error \\
\hline Duplicate patient & 9 & 1 & Yes & $\begin{array}{l}\text { (1) RIS-HIS failure } \\
\text { (2) Patient name change }\end{array}$ \\
\hline
\end{tabular}

Abbreviations: S, significant; L, incomplete information; I, inconveniece.

ing to two metrics. The first metric deals with the severity of potential adverse clinical impact of the error. The classifications are significant, incomplete information, and inconvenience. For example, if Ms Jones' image were mislabeled and assigned to $\mathrm{Mr}$ Smith, a significant error in the clinical management of $\mathrm{Mr}$ Smith could occur. Therefore, mislabeling of a patient image has been categorized as a significant error. An error is categorized as incomplete information if the information presented to the clinician is accurate but not complete. In the example above, the error resulting in Ms Jones' image being unavailable (as Ms Jones' image) is classified as incomplete information. The final category is labeled a clinical inconvenience. If an image is sent to the viewing station improperly oriented, it presents an inconvenience for the observer, but poses little risk of causing adverse clinical care to the patient. From Tables 2 and 3, $12.6 \%$ of the errors were categorized as significant $(1.02 \%$ of all images), $57.5 \%$ resulted in lost information (4.62\% of all images), and $29.9 \%$ were categorized as inconvenient $(2.40 \%$ of all images).

The second metric categorizes the error as unique to the PACS or common to both the PACS and conventional film management sys- tem. Of the 12 errors observed during this study, 8 occur in both the PACS and conventional film management system. Four errors were unique to the PACS system. Tables 2 and 3 include the values of this metric for each error type.

\section{DISCUSSION}

The cause of all errors found was investigated. The results of this investigation are listed in Tables 2 and 3. A flowchart of information passing through our ICU-PACS is shown in Fig 1. Sources of errors occurring in the system are highlighted. The majority of errors are attributable to technologist oversights ( 34 errors, $39.1 \%$ of errors) or HIS-RIS interface failures (33 errors, $37.9 \%$ of errors). Eighteen errors (20.7\%) were caused by a lack of radiologic interpretation available after 24 hours. CR or PACS hardware failures caused errors for two images (1 lost image, and 1 image with no flashcard). The ICU-PACS was designed to provide redundancy of all major equipment, with no single equipment failure resulting in the overall failure of the system. Previous documentation ${ }^{15}$ indicates that individual components of the ICUPACS system are available $\geq 97 \%$ of the time.

Table 3. Additional PACS Errors

\begin{tabular}{lcccc}
\hline \multicolumn{1}{c}{ Parameter } & Frequency & Impact on Information & Uniqueness to PACS & Cause \\
\hline Image processing & 1 & $\mathrm{~L}$ & Yes & Technologist error \\
No flash card & 1 & $\mathrm{~L}$ & Yes & CR flash card limitation \\
ADT error & 6 & $\mathrm{~L}$ & No & RIS-HIS failure \\
Repeated images & 3 & $\mathrm{I}$ & No & Technologist OC error \\
\hline
\end{tabular}

Abbreviations: $L$, incomplete information; $I$, inconvenience; ADT, admission, discharge or transfer. 


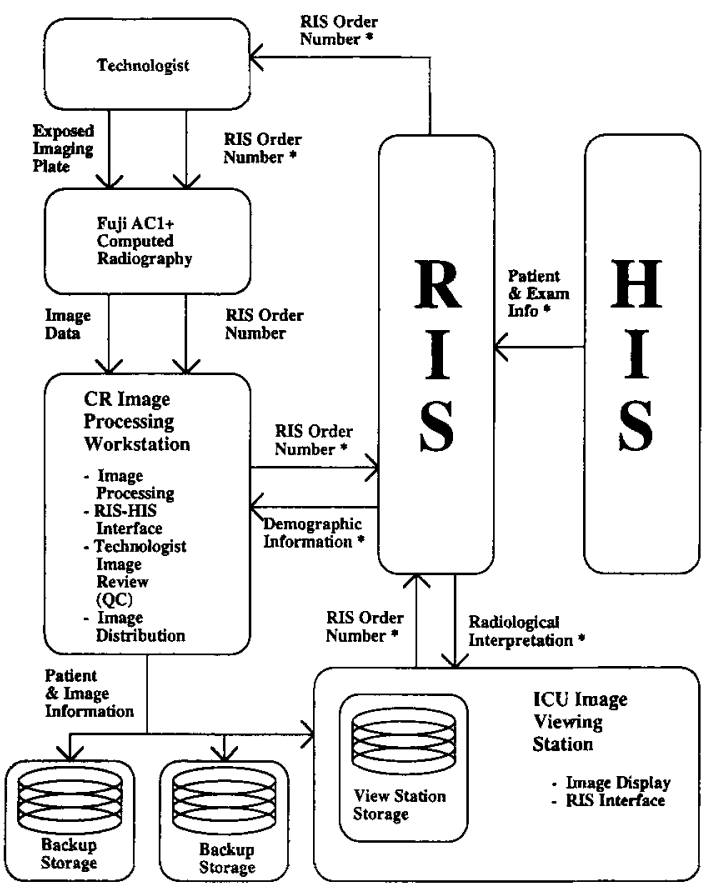

Fig 1. Diagram of information flow through the ICU PACS Interfaces between systems, with information flow direction, are indicated by arrows. Interfaces highlited with an asterisk are identified as causing the majority of QC errors.

The two mentioned hardware failures were the only instances where equipment failure (ie, CR device or computer failure) lead to delays in sending images to the ICU.

It is important to understand that a single failure can manifest itself as several errors. For example, an incorrectly entered order number results in wrong patient information being associated with an image. If the incorrectly entered order number corresponds to a patient in the MICU, this error would result an incorrect image being presented to a clinician, and classified as a severe error. Continuing our example, if the image was of a patient in the MICU, the incorrectly identified image would not be sent to the MICU. This would result in a lost image, which is categorized as incomplete data. Errors related to manual entry of order numbers can be corrected by implementing a less error-prone method of data entry. Bar coding of patient information onto study requisition forms ${ }^{17}$ and digital connection of CR systems to RIS terminals ${ }^{18}$ are currently being investigated.

The interaction between the HIS-RIS and the PACS is complicated. ${ }^{19}$ The ICU PACS requests patient demographic and report infor- mation from the RIS. The RIS, in turn, depends on the HIS for much of its information. Any disruption in the flow of information between the HIS, RIS and the ICU PACS is categorized as an HIS-RIS failure. Manual procedures exist, as part of the routine PACS operation, to correct these potential errors. However, there is no guarantee that the technologist will detect and correct, in time, errors associated with HIS-RIS failures.

The error "No order number" is frequently caused by an HIS-RIS failure. Investigation showed that this error is attributable, in most cases, to the current RIS and HIS data-backup procedures. The RIS is backed up (while online) daily between 8:00 PM and 8:30 PM. The HIS is backed up (while off-line) daily between midnight and 2:00 AM. These times are approximate, and may be extend as late as 5:00 AM for the HIS. Orders requested during backup periods must be entered after both systems become available. The ten examinations classified as "No order number" in Table 2 are shown in Table 4 with the corresponding time of day the examination was performed. The correlation between the occurrence of this error and the schedule of the RIS-HIS backup procedures is obvious.

One expected outcome of the ICU PACS implementation was a decrease in the number of lost ICU films and a corresponding improvement in reporting and billing of these examinations. Therefore, the problem of "No diagnosis" was investigated further. An image was labeled as "No diagnosis" if the radiologic interpretation was not available on the clinical viewing station 24 hours after the image was produced. Overall, 1,064 of the 1,082 images studied (98.3\%)

\begin{tabular}{lr} 
Table 4. Images Appearing on Clinical Viewing Station With \\
No Order Number \\
\hline Order No. & Time of Exam \\
\hline 429717 & $1: 11 \mathrm{AM}$ \\
426838 & $3: 20 \mathrm{PM}$ \\
431392 & $12: 48 \mathrm{AM}$ \\
444892 & $2: 56 \mathrm{AM}$ \\
444897 & $2: 58 \mathrm{AM}$ \\
445250 & $2: 09 \mathrm{AM}$ \\
Unknown & $1: 04 \mathrm{AM}$ \\
455421 & $7: 56 \mathrm{PM}$ \\
455405 & $7: 55 \mathrm{PM}$ \\
467740 & $12: 55 \mathrm{AM}$ \\
\hline
\end{tabular}


had radiologic interpretations available within 24 hours. Of those images without radiologic interpretations, one report was not available because of an HIS-RIS to PACS interface failure. In five unreported cases, the film was in the MICU and not available for radiologic interpretation. In three unreported cases the film was available for interpretation in the chest reading room, but no interpretation was available from the RIS. The status of the remaining nine images was unknown. In comparison, a recent departmental survey of 62 chest radiographs showed that 52 examinations $(83.9 \%)$ were transcribed within 25 hours.

It should be noted that there are several limitations to the study methodology discussed above. Errors were detected only when an MICU patient was involved. As the system expands, it is expected that number of different errors, and the frequency of known errors, will change. Additionally, if this study was expanded to encompass several viewing stations, overlap in reporting of errors would occur. For example, a mislabeled image for a patient in one ICU could show up on a viewing station in another ICU.

Rapid, reliable access to information plays an important role in maintaining high quality in a PACS. To achieve this requires simple, userfriendly interfaces for humans and robust interfaces between information systems. This issue is emphasized by the fact that every error type uncovered by this study, except for the flash card processing error and the loss of a single image, was preventable.

The ICU PACS system discussed relies on manually entered order numbers for the initial identification of patient images. This is not a desirable situation. The existing interface between the RIS-HIS and PACS is unreliable, as is the HIS-RIS system. This is the result of the current implementation of the interface (a terminal emulation) and the extended periods of time ( $>10 \%$ of the day) that the HIS-RIS is unavailable. Combined, these two factors account for 48 of the 87 errors $(55.87 \%)$ that occurred in our sample. It is strongly recommended that any site consideration implementing a PACS seriously consider the user and HIS-RIS-PACS interfaces early in the design and implementation stages.

A QC procedure that is performed by a technologist on every image before distribution to clinical review stations. Despite this measure, a significant number of errors go undetected. Thus, a separate check of information produced by a PACS is necessary. As a result of this study, the PACS has been modified and a technologist level position is being created to perform routine PACS QC.

\section{ACKNOWLEDGMENT}

We thank Drs H. Nath, R. Koehler, and L. Berland for their insightful comments during the preparation of this manuscript and Dr Wlad Sobol for his helpful comments and review of early drafts of the manuscript.

\section{REFERENCES}

1. Quality assurance for diagnostic imaging equipment: Recommendations of the National Council on Radiation Protection and Measurements Report 99. Bethesda, MD, National Council on Radiation Protection and Measurements (NCRP), 1988, p 4

2. Quality assurance for diagnostic imaging equipment: Recommendations of the National Council on Radiation Protection and Measurements Report 99. Bethesda, MD, National Council on Radiation Protection and Measure ments (NCRP), 1988, p 22

3. Stewart BK, Massoth RJ, Thomas SR: Mini-PACS, in Hendee WR, Trueblood JH (eds): Digital Imaging: Medical Physics Monograph No. 22. Madison, WI, Medical Physics Publishing, 1993, pp 123-156

4. Ravin CE: Initial experience with automatic image transmission to an intensive care unit using picute archiving and communication system technology. J Digit Imaging 3:195-199, 1990

5. Witt RM, Cohen MD, Appledorn CR: Initial experi- ence with a radiology imaging network to newborn and intensive care units. J Digit Imaging 4:39-42, 1991

6. DeSimone DN, Kundel HL, Arenson RL, et al: Effect of a digital imaging network on physician behavior in an intensive care unit. Radiology 169:41-44, 1988

7. Gillespy T, Staab EV, Lawrence E: Electronic imaging in a teaching hospital intensive care unit: Evaluation of the clinical review system. J Digit Imaging 3:124-128, 1990

8. Cho PS, Huang HK, Tillish J, et al: Clinical evaluation of a radiological picture archiving and communication system for coronary care unit. AJR 151:823-827, 1988

9. Parsons DM, Kim Y, Haynor DR: Quality-control of cathode-ray tube monitors for medical imaging using a simple photometer. J Digit Imaging 8:10-20, 1995

10. Halpern EJ: A test pattern for quality-control of laser scanner and charge-coupled-device film digitizers. J Digit Imaging 8:3-9, 1995 
11. Marsh DM, Cooney P, Malone JF: An assessment of the variation in image quality with multiformat cameras. Radiat Protection Dosimetey 57:277-280, 1995

12. Freedman M, Steller D, Jafroudl H, Mun SK: Quality control of storage phosphor digital radiography systems. J Digit Imaging 8:67-74, 1995

13. Seibert JA, Barnes GT, Gould RG (eds): Specification, Acceptance Testing and Quality Control of Diagnostic X-Ray Imaging Equipment. Woodbury, MA, American Association of Physicists in Medicine, 1994

14. Roehrig H, Yu T, Krupinski E: Image quality control for digital mammographic systems: Initial experience and outlook. J Digit Imaging 8:52-67, 1995

15. Tucker DM, Barnes GT, Koehler RE: Picture archiving communication systems in the intensive care unit. Radiology 196:297-304, 1995
16. Wandtke JC: Bedside chest radiography. Radiology 190:1-10, 1994

17. David G, Gregory S: Adding a microcomputer barcode network to a minicomputer-based radiology information system. J Digit Imaging 3:31-33, 1990

18. Frank MS, Rowberg AH, Tecotzky R: Integrating a radiology information system with a commercially-available computed radiology apparatus: An example of an interactive gateway, in Boehme JM, Rowberg AH, Wolfman NT (eds): Computer Applications to Assist Radiology. Carlsbad, CA, Symposia Foundation, 1995, pp 587591

19. Breant CM, Tiara RK, Huang HK: Interfacing aspects between the picture archiving and communication system, radiology information system, and hospital information system. J Digit Imaging 15:88-94, 1993 